\title{
Modelling Education Systems: An Ecological Approach
}

\author{
John Smith \\ University of Greenich, United Kingdom
}

\begin{abstract}
The specific aim of this paper is to outline some core, and developing concepts essential to the idea of the ecologies of education systems. The principle of self-organisation is recognised in sociology. But nothing organises itself in isolation; self-organisation is therefore re-conceptualised as auto-ecoorganisation. Similarly, self-reference is accepted in the human construction of meaning and understanding. The ecological perspective inserts this caveat: in order to survive, these constructions must be ecologically robust. A parallel re-conceptualisation is proposed: auto-exo-reference. Where social theory emphasises human uniqueness and autonomy, evolutionary psychology makes the more modest claim that humans have a greater degree of post-natal-plasticity. This means that preconscious dispositions, instinct, emotion and embodiment play a role at least as important as conscious intentionality. The implications of these issues for education research will be explored along with associated concepts such as inherent variance, path dependency and scale. Finally these concepts are put to use in sketching a model of the dynamics of education systems.
\end{abstract}

\section{Introduction}

In the recent past, the greater part of educational research and policy in the UK has been concerned with the delivery of the curriculum, issues related to inclusion and reducing discrimination, promoting the Third Way agenda and the routinely stated but unexamined connection between 'good' education outputs (meeting targets) and economic advantage. The Coalition is now proposing to experiment with a somewhat different relationship between the state and education providers. Alexander [1] has pointed to the absence of an overall philosophy of education for GB and exclusive concentration on delivery and measurement of standards. The research debate remains, in my view, a stalemate represented on the one hand by quantitative measures (SATs scores, attendance, reading ages, percentages of pupils scoring reasonable grades in public exams) - and on the other by 'constructivist' emphases by academics on the range of possible interpretation, cultural and ethnic perspectives, the 'active' individual leaner set against the prescriptions of curricular expectation.

This paper proposes a different perspective and, following the development of its basic concepts, a different set of methodologies. The former, at this stage, is necessarily more developed than the latter. My approach will be grounded in complexity theory and in the philosophy of ecology. The specific aim of this paper is to outline the following core, and developing concepts essential to the field of the ecologies of education systems. As such, I aim to contribute to both new directions and issues in educational research - but equally importantly, to promote the understanding of 'new' relevant disciplines and therefore the multidisciplinary outcomes of their interaction.

The following are presented serially. They are, however, necessarily recursive and consequent on each other. The order, then may appear arbitrary, or even variations on the same theme.

Finally the consequences for modeling education systems are examined.

\section{Self-organisation}

Durkheim proposed that social phenomena are sui generic. The standard social sciences model, SSSM [2] has emphasised the autonomy of human agency. Complexity theory has proposed this alternative. Nothing organises itself in isolation; the environment is both the enabling ground and limit of all such possibilities. Self-organisation is therefore reconceptualised as auto-eco-organisation [3, 4].

It is part of the ideological commitment of education systems, especially in Western democracies, that we humans are able to 'bootstrap' ourselves into ever-better intellectual and moral positions; that how we 'end up' is up to us - up to individual effort at selfbetterment. Similarly, we should as communities display the same kind of plasticity, improving our relationships to and understanding of others. This has 
been actively and rightly pursued in terms of, for example, gender, ethnicity, and (dis)ability.

At the same time, there is a marked tendency to attribute agency differentially to those conventionally seen as having power. Thus the accusation that schools have 'failed' whatever the current group causing concern - girls, black boys, working class white boys is commonplace. This position both grants agency to schools or teachers or 'the system' and reciprocally absolves, disempowers, exempts the disadvantaged group from any agency or responsibility for outcome. This denial of action, power, responsibility, selfdetermination would be unthinkable in many other areas of educational research: learning theory, children's voices and culture, ethnic identity.

I propose that this 'position' (if you can call it that) is contradictory, ad hoc, and more importantly, no credible or rational basis for educational research.

The problem, as I see it is an insufficient theoretical understanding of the ecology of self-organisation. First, self-organisation is not simply a social phenomenon; second the reciprocity of auto-ecoorganisation is obscured by the problematics of asymmetric power and the political criticism it generates.

Unless we propose a full retreat to Creationism, self-organisation is a pervasive property of the universe, the planet and the biosphere. Prigogine [5] has drawn attention to the processes of selforganisation in chemical systems far from thermal equilibrium. An example of this phenomenon is the self-organisation, ontogenesis and metabolism of the living. Kauffman [6] has published extensively on this in relation to the auto-catalysis of proteins, genetic interactions, ecologies, societies and markets. There are many other examples [7].

The consequence is that we cannot now call social phenomena sui generic in either the narrow Durheimian sense or the more radical propositions of creationists and constructionists. We must understand social phenomena as auto-eco-organisational. Put more bluntly, we can only self-organise (construct) to the extent that our past, present and future 'environment' permits. By 'environment' we mean everything that humans are networked with: the biosphere, our past, technology, information, others etc. As a methodological prescription for educational research, we must pursue precisely the question of what 'education' interacts with rather than treating it as a stand alone discipline. In this sense, the infertile squabble between the psychologists and the 'new' sociologists of education [8] must be abandoned. Both need to speak to each other. Our terror over the eugenic past of socio-biology and evolutionary psychology must also be suspended. There is nothing to fear in granting our relationships to other primates, nor our more general interconnection with the biosphere.

Perhaps I am over-stating the case here. There is something to fear. We have to face the limits of our own self-invention. The implication of sui generic social phenomena - the 'standard social science model' - that we author ourselves becomes modified. Evolutionary psychology proposes the more modest and plausible concept of high levels of post-natal plasticity as the distinctively human characteristic.

Which should we prefer: a concept of selfinvention that is by definition contingent and conventional; or a more grounded image of the human animal, capable of invention but still recognising human need? Which is the more appropriate prospect, especially for educational research whose primary subject remains the young and very young? Can we persevere with absurd position at the centre of our paradigm that childhood is an invention - that 'it' (whatever 'it' is) can be reinvented at will, infinitely otherwise? This is the inherent contradiction of the constructionist versions of education - a contradiction in which the questions of need and vulnerability cannot even be raised, except as 'constructions'.

I now turn to the asymmetry of auto-ecoorganisation. The paradigm case, for me, is the Holocaust. The Nazi regime claimed the absolute right to construct and ultimately deconstruct those of Jewish origin. In educational settings the position is less extreme and yet we persist in thinking about it in an unhelpfully asymmetric or extreme manner. On the one hand, we celebrate the agency of children and young people so far as their active learning and self identity is concerned. The idea that children are 'experts' in their own needs is routinely stated [8]. Ironically, this implies that children are in some sense socially exempt, if not wholly socially isolated. Common sense, ordinary politics, recognises at least a degree of adult responsibility. Any groups, who are only experts in themselves, are at extreme environmental risk.

On the other hand, schools routinely fail whoever they are seen to fail at the moment and those they fail are victims. They certainly bear no responsibility for outcomes where schools do the failing. Again, the failed group is at extreme environmental risk. I want to argue that neither position is credible because the known ecological pragmatics of interaction are disastrously ignored.

Humans are inherently social beings. The first and most fundamental level of agency is differential membership. This why schools fail - if they fail groups rather than individuals. The (sub)group is inherently active, simply through its identifiable existence. This why 'institutional' discrimination is so pervasive. Moreover, whatever the sub-processes education systems are geared toward the production of 
skills, expertise and shared values. Given this distinctive purpose and our commitment to meritocracy, mutual forms of discrimination are highly likely. For education systems, poor literacy is necessarily discriminated against. For those children and young people who find the normal curriculum dispiriting or alienating other recourses are bound to be attractive. Consequently, there is a level of active and intentional disruption in many schools because the perceived social benefit, however short term, is greater than conformity with the purposes of the curriculum. The UK is in collective denial about this, or at least of its scale. And yet the pragmatics of disconnected selfprotection at the individual [9] and the social level are well known.

\section{Self-reference}

It has long been argued that organisms and human cultures are self-referential in their construction of meaning and understanding. The ecological perspective inserts this caveat: in order to survive, these constructions must be ecologically robust. A parallel re-conceptualisation is proposed: auto-exoreference [10].

The social construction of meaning and selfreferential understanding is routinely taken by SSSM as a primarily if not uniquely human phenomenon. In fact the concept of self-relationality was pioneered and far more fully developed in cognitive theory by Maturana \& Varela [11, 4]. The key insight is that organisms are autopoietic (self-structuring) and this includes the ways they sense and respond to their environments. Rather than a relation of correspondence, organisms are seen to 'bring forth worlds' through being selectively 'structurally coupled' to their environment. So long as this relationship is sufficient - i.e. ecologically robust - the question of 'truth' is replaced by pragmatics. I want to argue that the constructionist perspective currently employed as part of SSSM lacks this important caveat. Humans are not simply constructors of meaning, but they do so within the confines of an environment containing both social and non-human imperatives. Interpretations that don't 'work' will not survive or will be extensively modified. Again we should understand the term 'work' as a matter of contexted sufficiency rather than correspondence.

Auto-exo-reference therefore presents important parallels with auto-eco-organisation. The result is a transformation, again, away from contingency and conventionality and toward embodied cognition. There is a parallel field division with semiotics and semiosis. Semiotics stresses the balance of arbitrariness of the signifier and relational conventionality. Semiosis stresses the embeddedness of signs in the biosphere.
The former is exemplified in the work of Saussure and Derrida and is well known to SSSM. The latter is associated with Pierce, Seboek and Deely, and 'embodied' philosophy is far more theoretically sophisticated but hardly known to SSSM. This is a situation that must be rectified [12].

A second parallel is the challenge presented in the context of self-organisation. Can we, after many centuries of privileging the human over the animal - an essentially monotheist conception - can we live with and theorise more grounded image of the human animal, capable of invention but still recognising human need?

\section{Post-natal plasticity}

Where the SSSM emphasises human autonomy, evolutionary psychology makes the more modest claim that humans have a greater degree of post-natalplasticity. This means that pre-conscious dispositions, instinct, emotion and embodiment play a role at least as important as conscious intentionality. This is being recognised in the recent regeneration of interest in learning theory [13].

The proposal of a far more modest notion of human post-natal-plasticity to replace the notion of human autonomy cannot be welcomed without reservation. I want to argue that we are quite able to live without the illusion of autonomy which is simply an idiom of the extremes of SSSM. And in making that concession we must rule out the despicable history of eugenics that can be - but should not be - associated with evolutionary psychology. The misgivings are easy to spot: feminists will fear renewed discrimination of the grounds of innate gender-dispositions; racism could be renewed on similar grounds. I haven't space to review the defences made by evolutionary psychology and suggest instead that you read Tooby and Cosmides' [2] spirited argument.

I want to raise what I regard as a more fundamental ontological question. The proposal of human autonomy appears at first hand to allow us greater freedom than that proposed by post-natal plasticity. This is not the case. The former 'allows' us, in theory at least, to construct ourselves but at the price of conventionality: it could always be otherwise. Put bluntly, who we choose to be is up to us, but it doesn't actually matter and we can't justify it anyway. Such arguments bedevil modern aesthetic philosophy. It would be ruinous if this kind of arbitrary assertion were to gain force in politics, or education. Yet, it is not far from possible. Can you, for example, make a spirited rebuttal of the human right to educate 'my' children as 'I' wish? Would you as an academic be comfortable with an enforced communitarian doctrine of education? Are you prepared to politically commit to something like 
The Third Way or New Schhools? Politicians are, of course.

I propose a different view. It is the limitation itself - the modest plasticity - that saves us from this impasse. The paradigm example is language itself. If human language did not have a certain universal character which interacts with its environments in universal ways, then the variety of human language would be self-organising on that fundamental ground, nor would they be translatable. Indeed, taken to its furthest, any communication would be, in principle, impossible. Many more examples may be generated. Let us take this general one: the basis of the organised variety of human cultures and technologies is grounded in human need. Otherwise they are literally groundless, have no bases nor impetus for organisation, no standards of human welfare by which they can be judged.

\section{Inherent variance}

Where particles and molecules have a more or less invariant character, organisms and ecologies, including human ecologies and social systems are trans-finite phenomena, capable of evolution and change. This is clearly an aspect of dynamic auto-eco-organisation.

Given the position in the previous section, needs, predispositions, 'nature' are the source and grounds, not the limits of variation. The human animal is particularly posed between necessity and invention. Dawkins [14] contrasted the need for genetic 'fidelity' with a corresponding but more open feature of iteration in human culture. Genes were contrasted with memes.

It is worth reconsidering the function of genetic information. Given the definitions offered by thermodynamic complexity theory systems far from equilibrium are in constant interaction with their environments. They are capable of, even driven to, evolving new forms of order. Living systems are also far-from-equilibrium but subject to stricter informational control through DNA. This 'intervention' of informational control in selforganisation is the signature of the living. Where informational control fails, the structure of the organism degrades.

It is crucial to understand the limits of genetic determinism. Where the repertoire of possible actions is limited (for example a spider making a web) the level of genetic determinism is high and the need for post-natal plasticity is minimal. For mammals the need to respond to a changing environment is such that high levels of post-natal plasticity are necessary to support an extensive repertoire of possible actions. Humans have evolved to the extreme of this spectrum. As Dennett [15] puts it: Freedom Evolves nicely catching the complex paradox we have to live with (literally!).
Put differently, the evolutionary path 'chosen' by humans minimises genetic determinism and maximises post-natal plasticity. Genetic determinism for humans, therefore, makes limited evolutionary sense. But this does not mean we have escaped our genetic heritage.

For example, instinct and emotion are recognised by many disciplines as pivotal to humanity [16]. They are taken as 'hard-wired' rather than learnt. SSSM does not readily concede this: the vast majority of behaviour is taken as learned or 'constructed'. But this misses a basic economy of survival. Instincts and emotions are fast and cheap in the sense that they do not need nor depend on prolonged deliberation. Indeed, that would be suicidal. Any competent human does not respond 'intellectually' to dog bark, a growl or the thrust of a snake, or an aggressive person with an interrogation of the precise meaning. That may follow but the instinct for self-protection comes first, without deliberation. In this sense, the insistence that all behaviour is learned is as one-dimensional and ecologically unsupportable as the idea that all human behaviour is genetically determined. This complex multidimensionality is our condition, not something we can avoid by reductions of either simple geneticism or simple rationalism.

Memetic iteration, on the other hand, takes place with cultures and in human minds. Whilst it cannot be simply classified as conscious (for example learning a language, taking on a dialect) it is not unconscious in the sense that (say) the immune system is. Sexual disposition on the other hand is taken by some as predisposition and others as learned. Either way, the social performance of gender has clear memetic components.

Memetic iterations are therefore not tied by genetic predisposition. They are inherently more tied to plasticity and experimentation. It is this propensity to intuit patterns of iteration that lies at the centre of our social identities and experimentations.

This interplay, it seems to me, is a far richer way of topicalising the production and performance of difference than the limited forms of constructivism. Within educational settings, it would provide for the kinds of modelling of ecological interaction that could draw on disciplines as (currently) diverse as developmental psychology, ethnography, social psychology, learning theory, cognitive science and sociology. Some of this is already implicit in the best work in education [17].

It is also evident in behavioural economics where, for example, the concept of self-signalling through the $\mathrm{I} / \mathrm{me}$ binary is crucial [18]. The problem is that it remains implicit in education theory and we are not talking to others whose disciplines overlap to a degree. If we better understand the processes of these sorts of pragmatics, we may be in a better position to ethically 
judge their outcomes and possibilities. We may, for example be able to distinguish those types of memetic iteration - i.e. patterned behaviours (in education) that are chaotic in the sense of having extremely limited immediate functions but without any robust ecological future.

\section{Path dependency and scale}

Where particles and molecules can be understood viably understood as single entities, (human) ecologies exhibit a patterned evolutionary past which profoundly influences their contemporary character. Moreover, this history demonstrates as a matter of fact a degree of ecological robustness such that breaking out of this path, then, poses formidable obstacles. Similarly, the dynamics of a system containing a small number of elements co-acting across a restricted area will be transformed where an increased number of elements produce more complex interactions. This combination of variation, path dependence and scale variance has been called a perfect storm [19]. However, the result is not chaos but complex patterning showing recurrence, 'attractors' and degrees of ecological viability.

\section{Preliminary Conclusions}

One conclusion is firm: this renewal of interdisciplinarity is far richer than the SSSM as the basic paradigm for educational research. Beyond that, we are still in a period of exploration. The task as this stage is to place these issues in the mainstream intellectual culture of (educational) research.

The second is also clear: reductionism versus 'thick' descriptions - in education this tends to present itself as the difference between psychological and sociological explanations respectively - is no longer sustainable. Instead we have a situation in which the 'ground' (for example the human individual) is a necessary but not a sufficient condition for subsequent emergent orders (for example, languages technologies, cultures, markets). The 'suppression' of either is ontologically indefensible.

On this basis, then, I suggest that the first requirement is the re-examination of the 'smeared' relationships, if I can use that term, between varying forms of structure and agency at both the individual and the collective level in all forms of social enquiry, especially educational research. In this 'smeared' reconceptualisation, customary 'gross' polarities should be put aside. Individuation is made possible through social interaction, ethnicity and gender are accomplished through micro-ethnicity and microgendering. Macro understandings such as class and national identity, the acceptance or rejection of a cultural pattern or educational aspiration are the outcomes, not the definitions of the micro-processes. Class, for example, does not 'cause' but instead emerges. This is a radical departure. Finally, we must take the opportunity that education research has traditionally offered - but we usually decline - that is, a thoroughgoing commitment to inter-disciplinarity.

\section{From Interdisciplinary Theory to Specific Modelling.}

With these preliminary conclusions in mind, we can begin to sketch the task of modelling (an) education system(s). Imagine a simple mechanical or electrical system of human design. There is an input of some sort, a process of translation or modification, leading to an output. So far as this is designed the basic principle is that a given input is transformed into the desired output. Further, variations in the type or quantity of input should result in a related and proportionate change in output. Despite contrary indications and common beliefs, education is not that type of system.

Education systems, like economies and ecologies are composed of multiple agents (or 'designers' if you will, though term becomes ever more tenuous) and the outcome is determined probabilistically through their interactions. Differently put, outcomes are selfemergent. Who can decide whether outcomes are nonfunctional? 'State' education grants that decision to the government but that is legalistic, rather than factual. 'Private' education concedes that the market will decide but not that the outcomes will necessarily match individual aspirations.

The first two propositions of this model of education systems are then:

1. There is a multiplicity of 'contracted' agents or interested stakeholders.

2. Outcomes are self-emergent reflecting the inherent scale, variance and internal complexity of the system and do not necessarily concur with interests or intentions of particular stakeholders.

The simple input-transformation-output model described above does not describe the internal complexity of intention, agency and emergent outcome in ecological systems. But there is another dimension of difference, this time from the perspective of externality. The simple system delivers its product reliably and proportionately due to its isolation from its immediate environment. Think of the sheer investment in insulation that characterises a motor car: from paint to fuel purity, from springs, dampers and tyres to road construction. Any missing element and functionality 
ceases. Complex 'ecological' systems on the other hand are open to their environments to the degree that it becomes difficult if not impossible to distinguish between system and environment. And this 'entanglement' is not detrimental to functionality but is rather its condition. For example, are pupils 'internal' parts of the system or part of the environment in which the system works, or the raw material, or indeed, the principal agent? This again underscores the inherent variance of actual relationships.

The third and fourth propositions are then:

3. These systems are both internally and externally complex and open to their environments to such a degree that the boundaries are 'smeared'. Their identity is given in and by the smear. Or:

4. Education systems are auto-eco-organisational which is radically different from autopoiesis in the self- replicative sense developed by Maturana \& Varela (20). Luhmann (21) and others (22) are mistaken in employing the latter term for heterodynamics.

Education systems emerge through the interaction of qualitatively different 'agents', substrates, forces. So far as these are developmental in the biological or psychological sense, it would be wrong to take them as simply social constructs. So long as physical limits and technologies - for example cities and transport systems - are entangled in education systems, these factors have 'agency' as well in the sense of shaping and separating the possible from the impossible. Another clear shaping and limiting dimension is cost and resource availability. Simple social constructionist perspectives omit these considerations. Moreover, social constructionist are negligent of both ecology and path dependency. Put differently the possibility of (social) construction is constrained by past and present social construction as well as non-social obstacles.

Propositions five and six then:

5. Unlike autopoietic systems, auto-eco-organised systems consist of a number of qualitatively different 'active' dimensions, according to the specific history of self-emergence.

6. The notion of social construction must admit that it can operate or carries responsible only to $a$ degree and according to the actual elements of path dependency. Constructionist perspectives routinely fail to recognise that there is a world of difference between construction per se and constructing different responses to a precondition or habitual state (even if we end up with a new understanding of the precondition).
Nevertheless, education systems are perceived to have, or tend to have an over-riding stated purpose: the transmission and acquisition of knowledge. There are many nuanced approaches to this problem. For example, MacNaughton (23) identifies three 'regimes': one of conforming to, of reforming and of transforming society. The first seeks to reproduce knowledge and culture, the second to open it to reform through negotiated teaching and learning, the third to make radical transformations of power relations. It is of course possible that reforms and transformations become the new orthodoxy to be reproduced.

Others, such as Illeris (24), Jarvis (25) have stressed the activity as opposed to the passive acquisition of learning, its social contexts, costs and the associated influences, for good or ill, on identity. This is again a fundamentally ecological approach suggesting conflicts, alliances or a landscape of the more and the less possible. Prominent in these discussions is the idea that the knowledge transmission / acquisition process is beset by costs and contention. A maths lesson, for example, might be seen as the development of a normative corpus of knowledge, as the scene for individualised processes, some of which may be more viable than others, as a waste of time, as an occasion to humiliate a teacher or an 'opposed' pupil group. It is within contested psycho-social settings such as these that (special) educational needs become manifest Proposition 7 and 8, then

7. Even at the specific level of knowledge transmission / acquisition, outcomes are emergent and potentially unpredictable. Rather than simply designated a priori, they are self-referential in a pluralistic sense. Stated in the previous terms, they are auto-eco-referential. The 'auto' aspect of this reference will contain instinctive or emotional elements that are not necessarily subject to rational control. The 'eco' element will generate elements of reflection on how 'I' (can) appear and (afford) to operate with respect to others. They will be a degree of plasticity in this $\mathrm{I} / \mathrm{me}$ signalling, not autonomy.

8. Nevertheless, the intended outcomes routinely serve to measure the success or failure of both pupils and schools. This is a necessarily discriminatory reflection which reflects the routinely suppressed but necessary fact that outcomes do not correspond precisely to predictions: a 'degree of freedom' is always manifest.

The simpler social constructionist models are not open to this difference. Therefore the model of social solidarity they offer is limited. Using the example above, there is a clear distinction of authority 
(authority to construct) where the intended outcomes claim greater and juridical rights over and above the unintended. In the language of TenHouten (26) this type of solidarity presents itself as intensely hierarchical or agonic. The alternative form of solidarity displays negotiated competitive processes and is termed hedonic. In agonic solidarity the rights of the lower ranks of the hierarchy are withdrawn. The most extreme form is the withdrawal of rights over the lower individual's body or freedom. Schools present a history of this type of solidarity in the form of punishments and sanctions. They form the subject matter, explicitly or not, of neo-Foucauldian and other control theorists such as Olssen (27). What they lack is the clear structural distinction between the agonic \& hedonic and its emotional complexity in 'practising' solidarity. In particular, they seek to promote competitive equality (hedonic solidarity) without conceding the structural difficulties this promotes. Thus, the more radical forms of children's rights discourse, or the idea that children are experts in their own needs, for good reasons minimizes the more repressive aspects of agonic solidarity; but then ironically and unintentionally marginalises children by tacitly withdrawing them form the full range of social ecology. This is both unjustifiable theoretical simplification and isolation.

Propositions 9 and 10, then:

9. The structural imposition of agonic and the negotiation of hedonic forms of solidarity should be explicit requirements in modelling the socioecology of education systems. Agonic forms are clearly intended to limit the plasticity and plurality of possible outcomes.

10. The distinction agonic / hedonic is not fixed. It's dynamism in the $19^{\text {th }} \& 20^{\text {th }}$ Centuries presented highly centralised forms of legal sanction. It is open to question whether in the $21^{\text {st }}$ Century this legal relationship is giving way to a more clientoriented form - or whether regulation is simply taking a more devolved but widespread form. The implications for research, particularly into the question of governance are far reaching.

\section{Conclusion}

I have suggested that we re-examine the core subject matter with which we approach the question of education 'systems'. They may appear strange but this is only a question of habituation. The material is there to draw upon \& to develop. I have further suggested a series of propositions, by no means exhaustive, that we may consider in modelling the dynamics of education systems. My contention is that existing models are too simple. No doubt the rejoinder will be that mine are to complex to be practical.

My counter argument is this. Those at the sharp end of education: children, teachers, parents, administrative and ancillary staff and, yes, politicians - deal on a daily basis as a matter of course with the actual dynamic complexities of education systems. We cannot, as academics, continue to provide them with a simple, idealised abstraction that bears scant relation to a complex reality. That is not pragmatism, but practised ignorance.

I have argued that outcomes may be, to a degree, unpredictable or not congruent with original intentions. Nevertheless, this disparity can be read. That is why those 'in the field' can, and routinely do, claim better understanding. My contention is that it is not beyond academics to also engage these complex dynamics. Finally, we are not in a situation suitable for deterministic descriptions. A given input will not reliably nor proportionately lead to an expected output. The determinism of a 'positivist' standpoint is ontologically inappropriate. Nor are we adrift on an unstructured void occupied by conventional and arbitrary construals. The indeterminacy of unfettered human construction is equally ontologically inappropriate. We should instead speak of an ontology in which the space of the possible is spontaneously explored through its own actualisations. That would accord with the best and more recent ontologies in the process sciences. (28) We are part of that exploration.

\section{References}

[1] Alexander, R. (2009) Introducing the Cambridge Review, Esmee Fairbairn, Cambridge.

[2] Tooby, J. and Cosmides, L. (1992) 'The Psychological Foundations of Culture', in J. Barkow, J. Tooby, \& L. Cosmides, (eds.), The Adapted Mind: Evolutionary Psychology \& the Generation of Culture, Oxford University Press, Oxford.

[3] Morin, E. (2002) 'The Notion of the Subject' in D. Schnitman \& J. Schnitman (eds), New Paradigms in Culture \& Subjectivity, Hampton, New Jersey.

[4] Smith, J. and Jenks, C. (2006) Qualitative Complexity, Routledge, London \& New York.

[5] Prigogine, I. and Stengers, I. (1984) Order out of Chaos, Bantam, New York.

[6] Kauffman, S. (2008) Reinventing the Sacred, Basic Books, New York.

[7] Delanda, M (1999) 'Immanence and Transcendence in the Genesis of Forms', in I. Buchhanan (ed.), A Deleuzian Century, Duke University Press, Durham NC. 
[8] James, A. and Prout, A. (2005) (eds.), Constructing and Reconstructing Childhood: Contemporary Issues in the Sociological Study of Childhood, UK Falmer Press, London.

[9] Sims, P. (2000) Reasons \& Remedies, Mortimore Books, London.

[10] Morin, E. (2002) 'The Notion of the Subject' in D. Schnitman \& J. Schnitman (eds), New Paradigms in Culture \& Subjectivity, Hampton, New Jersey.

[11] Maturana, U. \& Varela, F. (1980) Autopoiesis and Cognition, D. Reidell, Dordrecht.

[12] Deely, J., (2003) 'The Quasi-Error of the External World’, Cybernetics and Human Knowing, 10(1), p. 29.

[13] Illeris, K., (2009) Contemporary Theories of Learning, Routledge, London and New York.

[14] Dawkins, R. (1976) The Selfish Gene, Oxford University Press, Oxford.

[15] Dennett, D. (2003) Freedom Evolves, Alan Lane, London.

[16] TenHouten, W. (2007) A General Theory of Emotions and Social Life, Routledge, Oxon.

[17] Evans, G. (2007) Educational Failure and Working Class White Children, Palgrave MacMillan, London.

[18] Lowenstein, G. (2007) Exotic Preferences:Behavioural Economics and Human Motivation, Oxford University Press, Oxford.

[19] Reiners, W. A. and Lockwood, J. A. (2007) Philosophical Foundations for the Practices of Ecology, Cambridge University Press, Cambridge.

[20] Maturana, U. and Varela, F. (1980) Autopoiesis \& Cognition, Dordrecht: D. Reidel.

[21] Luhmann, N. (1995) Social Systems, Stanford: Stanford University Press.

[22] Koshinen, K. (2010) Autopoietic Knowledge Systems in Project-Based Companies, Basingstoke: Palgrave Macmillan.

[23] MacNaughton, G. (2003) Shaping Early Childhood, Maidenhead: Open University Press

[24] Illeris, K. (2006) How we Learn, London, Routledge.

[25] Jarvis, P. (2006) Human Learning, London: Routledge.

[26] TenHouten, W. (2007) A General Theory of Emotions \& Social Life, Routledge, Oxon.

[27] Olssen, M. (2004) Culture \& Learning, Connecticut Information Age Publishing, Greenwich.
[28] Shaw R. and Turvey, M. (1999) 'Ecological Foundations of Cognition' in Nunez, R \& Freeman, W. (eds) Reclaiming Cognition, Bowling Green $\mathrm{OH}$ : Imprint Academic. Or: Kaufmann, S. (2008) Reinventing the Sacred, Basic Books, New York. 